Original Article

\title{
The effects of transcutaneous neuromuscular electrical stimulation on the activation of deep lumbar stabilizing muscles of patients with lumbar degenerative kyphosis
}

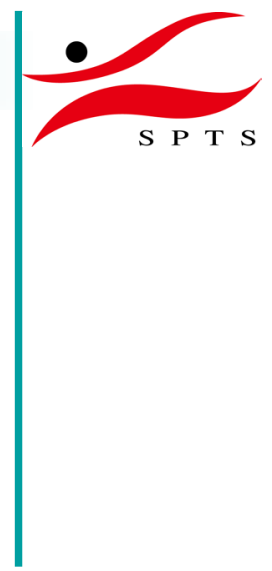

So Yeon Kim, $\mathrm{PhD}^{1)}$, Jin Hyun Kim, MD ${ }^{2)}$, Gil Su Jung${ }^{2)}$, Seung OK Baek, $\mathrm{MD}^{2)}$, Rodney Jones, $\mathrm{MD}^{3)}$, SAng Ho Ahn, MD, $\left.\mathrm{PhD}^{4}\right)^{*}$

1) Department of Biomedical Engineering, College of Medicine, Yeungnam University, Republic of Korea

2) Department of Physical Medicine and Rehabilitation, College of Medicine, Yeungnam University, Republic of Korea

3) Department of Anesthesia, University of Kansas School of Medicine, USA

4) Department of Rehabilitation Medicine and Spine Center, Yeungnam University College of Medicine: 317-1 Daemyung-Dong, Nam-Gu, Daegu 705-717, Republic of Korea

\begin{abstract}
Purpose] To investigate the effectiveness of three different neuromuscular electrical stimulation (NMES) protocols for the deep lumbar stabilizing muscles of patients with lumbar degenerative kyphosis (LDK). [Subjects and Methods] Twenty patients with LDK were recruited. Three stimulation protocols were investigated: stimulation of the abdominal muscles (protocol A); stimulation of the lumbar muscles (protocol B); and simultaneous stimulation of the abdominal and lumbar muscles (protocol $\mathrm{A}+\mathrm{B})$. Images of the obliquus externus $(\mathrm{OE})$, obliquus internus (OI), transversus abdominis (TrA), and lumbar multifidus (LM) muscles were captured by realtime ultrasound imaging (RUSI). [Results] The thickness of LM was significantly greater during stimulation than at rest for all three protocols. Thicknesses of the abdominal muscles (TrA, OI, and OE) were significantly greater during stimulation than at rest for protocols $\mathrm{A}$ and $\mathrm{A}+\mathrm{B}$. Thickness increases in $\mathrm{LM}$ were significantly greater during protocols $\mathrm{B}$ and $\mathrm{A}+\mathrm{B}$, but not during protocol $\mathrm{A}$. Thickness increases in the abdominal muscles (TrA, OI, and $\mathrm{OE}$ ) were significantly greater during protocols $\mathrm{A}$ and $\mathrm{A}+\mathrm{B}$, but not during protocol $\mathrm{B}$. [Conclusion] NMES can significantly activate the deep lumbar stabilizing muscles of patients with LDK. Protocol A+B of NMES is recommended to aid postural correction and low back pain (LBP) in patients with LDK.

Key words: Neuromuscular electrical stimulation, Lumbar degenerative kyphosis, Deep lumbar stabilizing muscle
\end{abstract}

(This article was submitted Sep. 14, 2015, and was accepted Oct. 30, 2015)

\section{INTRODUCTION}

Lumbar degenerative kyphosis (LDK) is a common cause of lumbar spinal deformity in Asian women aged 40 years and older. It is characterized by sagittal imbalance, a stooped posture and chronic low back pain (LBP). Associated with LDK is a loss of the normal lumbar spinal lordosis ${ }^{1-3)}$. Marked atrophy of lumbar extensors and degenerative changes in the lumbar spine are hallmarks of this condition. It is postulated that endemic lifestyles such as prolonged crouching, often required of agricultural workers, may be a factor in the development of $\mathrm{LDK}^{1-3)}$. In studies utilizing computed tomography (CT) and magnetic resonance imaging (MRI), extensive degenerative atrophy and fatty infiltration of lumbar extensor muscles have been reported ${ }^{1-3)}$. Moreover, in a state of sagittal imbalance, kyphosis or loss of lordosis of the lumbar spine can lead to LBP

\footnotetext{
*Corresponding author. Sang Ho Ahn (E-mail: spineahn@ynu.ac.kr)

C2016 The Society of Physical Therapy Science. Published by IPEC Inc.

This is an open-access article distributed under the terms of the Creative Commons Attribution Non-Commercial No Derivatives (by-nc-nd) License $<$ http://creativecommons.org/licenses/by-nc-nd/4.0/>.
} 
and various spinal disorders ${ }^{4-7)}$.

LBP is known to arise from multiple factors, including the loss of lumbar spinal stability ${ }^{8-11)}$. If a state of lumbar instability is not appropriately resolved, chronic recurrent LBP might occur ${ }^{12}$. Patients with LBP do not sufficiently activate the deep lumbar stabilizing muscles, such as, the lumbar multifidus (LM), transversus abdominis (TrA), and obliquus internus (OI) muscles, which are essential for lumbar spinal stability ${ }^{13-15)}$. Therefore, specific exercises for lumbar stabilizing muscles are essential in the rehabilitative strategy for patients with $\mathrm{LBP}^{16-20}$. However, traditional exercises for deep lumbar stabilizing muscles tend to be laborious and time intensive for therapists and patients. Since deep lumbar stabilizing muscles are smaller in patients with chronic LBP and contain a higher proportion of fat ${ }^{3}$ ), affected individuals find it more difficult to sufficiently activate these muscles.

Neuromuscular electrical stimulation (NMES) is a form of lumbopelvic stabilizing rehabilitation that is being increasingly used to reinforce muscle strength and prevent muscle atrophy ${ }^{18-25}$. This technique has also shown promise in training of the deep lumbar stabilizing muscles ${ }^{22-25}$. In addition, muscle activation by NMES has been reported to be significantly related to clinical improvements in patients with $\mathrm{LBP}^{24,26)}$. However, no report to date has been issued on the effects of NMES on the deep lumbar stabilizing muscles of LDK patients, and few studies have attempted to define the optimal protocol for the stimulation of deep lumbar stabilizing muscles by NMES.

In this study, the ability of NMES to stimulate the deep lumbar stabilizing muscles of LDK patients was investigated, using real-time ultrasound imaging (RUSI).

\section{SUBJECTS AND METHODS}

Twenty patients with symptomatic sagittal imbalance due to LDK, who visited the rehabilitation department of Yeungnam University Hospital, were recruited (19 females, 1 male, overall mean age $66.22 \pm 6.55$ years, range 54-74 years). The selected patients exhibited the characteristic clinical features of LDK, that is, a stoop with walking difficulties, an inability to lift heavy objects, difficulty walking on an inclined plane, and the need for elbow support when standing for any length of time. Whole-spine lateral views showed loss of lower lumbar lordosis resulting in retroversion of the pelvis with thoracolumbar lordosis compensating for sagittal imbalance. Patients with a history of osteoporotic compression fracture, spine surgery, spinal disease (trauma, tumor, scoliosis, Scheuermann's kyphosis, or stenosis), hip joint disease, or contraindications for NMES (pacemaker, edema, paresthesia, thromboembolism etc.) were excluded. The participants were provided with comprehensive oral and written information about all aspects of this study, and all provided their written consent to participation. This study was conducted prospectively and the Institutional Review Board of our hospital approved the study protocol.

Abdominal electrical stimulation was delivered through a set of 4 hydrogel surface electrodes $(5 \mathrm{~cm} \times 5 \mathrm{~cm})$ located on both sides of the anterolateral abdominal wall. Two surface electrodes were placed on the right and two on the left sides. The reference points were located $1 \mathrm{~cm}$ superior to the iliac crests on the mid axillary lines. Abdominal stimulation points were located $2 \mathrm{~cm}$ superior and $2 \mathrm{~cm}$ medial to the anterior superior iliac spines (Fig. 1) ${ }^{7}$. Lumbar electrical stimulation was also delivered through a set of 4 hydrogel surface electrodes $(5 \mathrm{~cm} \times 5 \mathrm{~cm})$ positioned bilaterally at an inter-electrode spacing of $\sim 2 \mathrm{~cm}$ on a cross line drawn on the L4-5 interspinous process (Fig. 1 ${ }^{28)}$. Stimulation pulses were generated using a portable research-stimulator (CMMX-001A; Cybermedic Corp., Republic of Korea), which delivered a constant current and a symmetrical biphasic waveform. Bi-phasic symmetrical pulses of $200 \mu$ s with an interpulse delay of $100 \mu$ s were employed. These pulses were delivered via the four surface electrodes at a frequency that produced tetanic isometric contractions of the targeted muscles $(50 \mathrm{~Hz})$. The overall contraction-relaxation cycle used was as follows: ramp up for 1 second, contraction for 8 seconds, and ramp down for 1 second followed by relaxation for 10 seconds. The subjects and investigators controlled the current intensities. The subjects were instructed to use the unit at an intensity that elicited maximum muscle contraction without discomfort, that is, no burning sensation or severe tetanic pain. The mean current intensity was $45.39 \pm 10.24 \mathrm{~mA}$ for the abdominal muscles, and $58.34 \pm 12.97 \mathrm{~mA}$ for the lumbar muscles. Each muscle was evaluated during three different NMES protocols: protocol A, stimulation of abdominal muscles, protocol B, stimulation of lumbar muscles; and protocol $\mathrm{A}+\mathrm{B}$, concurrent stimulation of abdominal and lumbar muscles.

A RUSI (Logiq 6 expert, GE Healthcare, UK) unit was used to measure changes in the abdominal and lumbar muscle

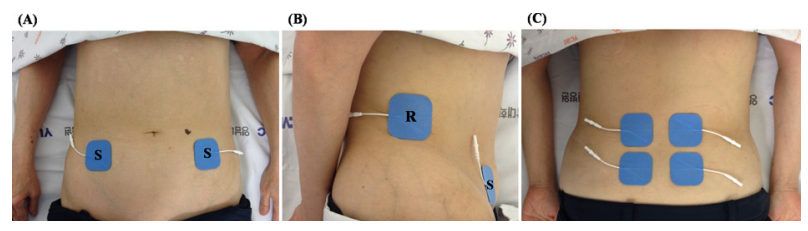

Fig. 1. Sites of stimulation used on the abdominal wall and lumbar paraspinal region (A) abdominal wall (frontal view), (B) abdominal wall (lateral view), (C) lumbar paraspinal region S: stimulation electrode, $R$ : reference electrode 
[TrA, OI, obliquus externus (OE), and LM] thicknesses during NMES of each protocol. To assess abdominal muscles, with subjects comfortably positioned supine with a pillow placed under the knees, a $5 \mathrm{MHz}$ curvilinear ultrasound probe was placed transversely across the abdominal wall along the line midway between the inferior angle of the rib cage and the iliac crest. The medial edge of the probe was placed approximately $10 \mathrm{~cm}$ from the midline, and, its position was adjusted to ensure that the medial edge of the TrA was $\sim 2 \mathrm{~cm}$ from the medial edge of the ultrasound image when the subject was relaxed (Fig. 2) ${ }^{29)}$. The location of the probe was marked to ensure its identical placement for all measurements. Static ultrasound images were captured at rest and again when maximum NMES stimulation had been reached. During image capture at rest, the subjects were instructed to breathe normally and evenly and an image was captured at the end of exhalation. During stimulation image capture, the subjects were instructed to hold their breath at the end of exhalation. Preliminary analysis of the results indicated that the right and left sides contracted in similar ways, and thus, further analysis was carried out using right side images. To evaluate the LM, the subjects were placed prone, with the abdomen supported as needed to ensure no more than $10^{\circ}$ of lumbar lordosis, and then instructed to breathe normally and evenly. Static rest images were captured at the end of exhalation (Fig. 3), and static ultrasonic images of the LM muscle were taken during each NMES stimulation protocol. All the above tasks were performed three times, and the average of the three measurements was used in the analysis.

Stored images were analyzed using ultrasound image measurement software. Muscle thicknesses were measured by an expert ultrasonogropher blinded to the image identities. On abdominal images, a grid was placed over the images and the thicknesses of OE, OI, and TrA muscles were measured at three sites, that is, at the image midline and at $1 \mathrm{~cm}$ either side of the midline. Cursors were placed on the superficial and deep boundaries of the muscles at the edges of the hypoechoic regions representing the locations of fascial separations between muscles ${ }^{29}$. On lumbar images, the thickness of the LM muscle was measured between the posterior-most portion of L4/L5 facet joints and the fascial planes between the muscles and subcutaneous tissues ${ }^{30}$. The average of the three measurements was used in the analysis and the effects of NMES were investigated using the changes in thickness between rest and during NMES (Figs. 4 and 5).

Statistical analysis was performed using the Statistical Package for the Social Sciences (SPSS) version 18.0 (SPSS, Chicago, IL, USA). The paired t-test was used to determine the significances of thickness differences at rest and during NMES for each muscle (LM, OE, OI and TrA), and one-way analysis of variance (ANOVA) and the Kruskal-Wallis test were used to analyze differences between the A, B and A+B protocols for each muscle. When a significant difference was found between groups, Tukey's post-hoc test was used for assess pair-wise comparisons. Statistical significance was accepted for $\mathrm{p}$ values $<0.05$.

\section{RESULTS}

The mean age, height, weight, and body mass index (BMI) of the twenty participants were $66.22 \pm 6.55$ years, 157.92 $\pm 8.66 \mathrm{~cm}, 57.94 \pm 6.75 \mathrm{~kg}$, and $23.27 \pm 2.42 \mathrm{~kg} / \mathrm{m}^{2}$. Significant thickness changes in the superficial and deep LMs were observed during NMES during all three protocols $(\mathrm{p}<0.05)$. Significant thickness changes in abdominal muscles (OE, OI, and $\operatorname{Tr} \mathrm{A}$ ) were also observed during NMES during protocols $\mathrm{A}$ and $\mathrm{A}+\mathrm{B}(\mathrm{p}<0.05)$, but not during protocol $\mathrm{B}$ (Table 1 and Fig. 4).

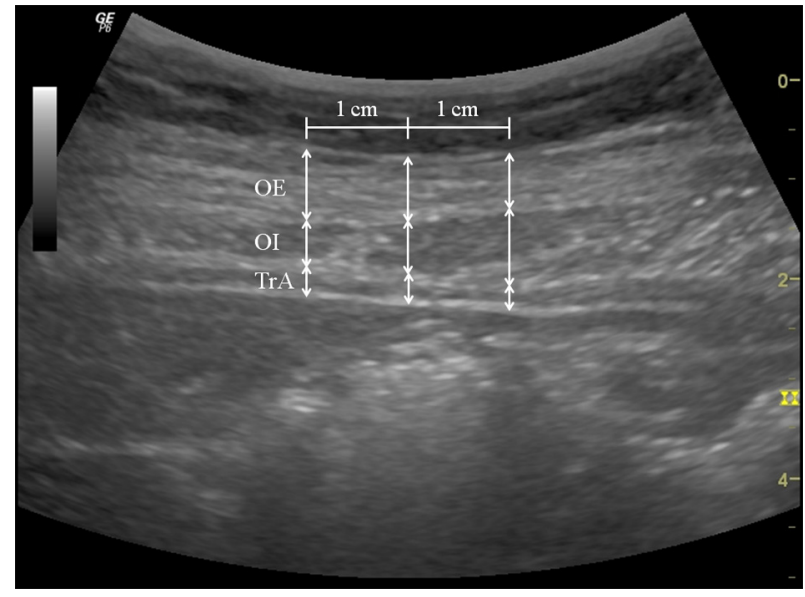

Fig. 2. Thicknesses of abdominal muscles during NMES Three vertical lines were drawn: one at the midline of the image and two $1 \mathrm{~cm}$ (adjusted for scale) either side of the midline. OE: obliquus externus muscle, OI: obliquus internus muscle, TrA: transversus abdominis muscle

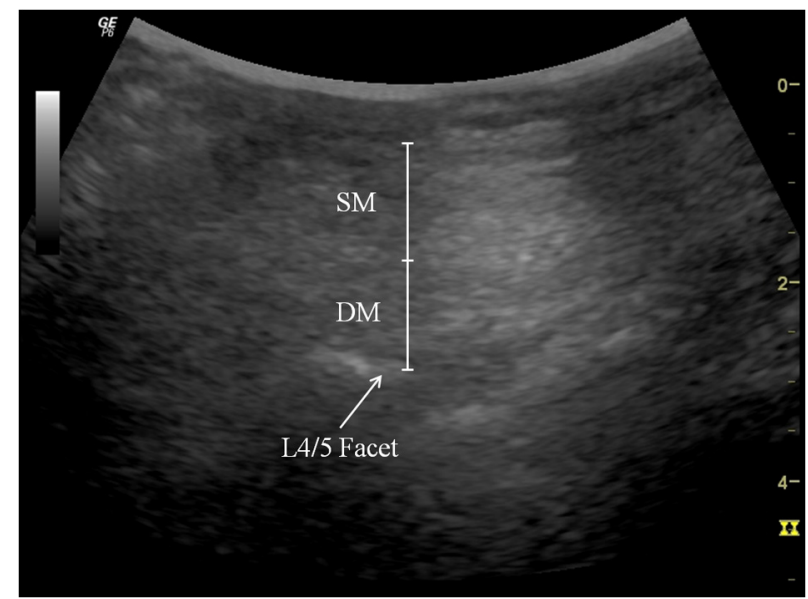

Fig. 3. Thickness of the lumbar multifidus muscle during NMES The lumbar multifidus muscle thickness was measured at the distance between the posterior-most portion of the L4/L5 facet joint and the fascial plane between the muscle and subcutaneous tissue. SM: superficial lumbar multifidus muscle, DM: deep lumbar multifidus muscle 
Increases in the thicknesses of the superficial and deep LMs during protocol B and A+B were significantly larger than during protocol A $(\mathrm{p}<0.05)$, but no significant difference was found between protocols $\mathrm{B}$ and $\mathrm{A}+\mathrm{B}$. Increases in the thicknesses of OE, OI, and TrA were significantly greater during protocols $\mathrm{A}$ and $\mathrm{A}+\mathrm{B}$ than during $\mathrm{B}$ protocol $(\mathrm{p}<0.05)$, but no significant difference was found between protocols $\mathrm{A}$ and $\mathrm{A}+\mathrm{B}$ (Table 2 and Fig. 5).

\section{DISCUSSION}

This study showed that the deep lumbar stabilizing muscles of patients with LDK were significantly activated by NMES. Furthermore, our results show the protocols B and A+B maximally stimulated the superficial and deep LM muscles, and that protocols $\mathrm{A}$ and $\mathrm{A}+\mathrm{B}$ maximally stimulated the abdominal (TrA, OI and $\mathrm{OE})$ muscles. These observations show that the deep lumbar stabilizing muscles (LM, TrA and OI) were significantly and maximally stimulated by protocol A+B. Previous studies have reported that NMES effectively activates the deep lumbar stabilizing muscles ${ }^{22-25)}$, and the results of this study confirm these findings. All studied lumbar and abdominal muscles, including the deep and superficial muscles, contracted simultaneously during NMES. These findings suggest that NMES may provide a means of strengthening the deep lumbar stabilizing muscles of patients with LDK. The possibility of contracting and thereby training the smaller poorly conditioned lumbar stabilizing muscles of patients with LDK is encouraging and may provided an additional avenue for rehabilitation.

Several studies have reported that NMES effectively activates the deep lumbar stabilizing muscles ${ }^{23,24,27,28)}$. Coghlan et al. ${ }^{23)}$ demonstrated a significant increase in TrA thickness during belt-type NMES of healthy subjects. In another study by the same authors ${ }^{24}$, patients with chronic LBP were found to show significant increases in the thicknesses of TrA, OI, and LM during loading tasks after 6 weeks of NMES intervention. Baek et al. ${ }^{28)}$ reported that 20 healthy subjects showed significant thickness increases in LM, TrA and OI during NMES of the lumbar paraspinal region, and Baek et al. ${ }^{27)}$ reported 20 healthy subjects showed significant TrA and OI thickness increases during NMES applied to the abdominal wall. Our results are in agreement with these results and suggest that NMES could be used to train the deep lumbar stabilizing muscles of patients with LDK.

Surgical treatment may be considered for LDK patients having difficulties performing activities of daily living due to severe sagittal imbalance. However, as the proportion of elderly individuals in the population has increased, the average age at the time of surgery has also increased, increasing surgical risks and complications. In addition, some patients complain of recurrent chronic LBP and sagittal decompensation related to posture even after surgical correction of kyphotic spinal deformities ${ }^{2,31)}$. Exercises that help train lumbar stabilizing muscles are typically adopted for conservative treatments of chronic LBP and the correction of kyphotic postures ${ }^{16-20,32)}$. Patients with LBP cannot sufficiently activate the deep lumbar

(A)

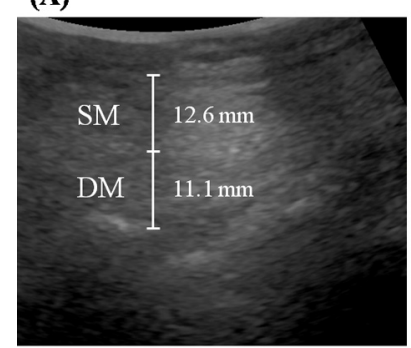

(C)

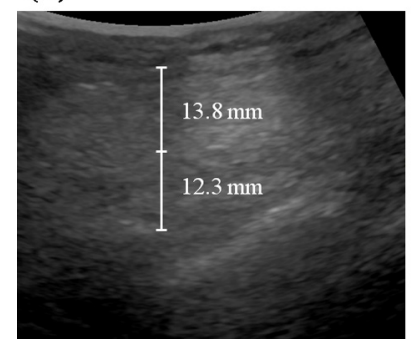

(B)

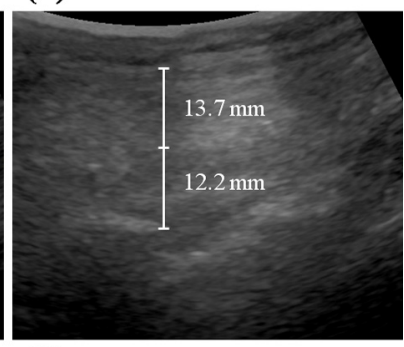

(D)

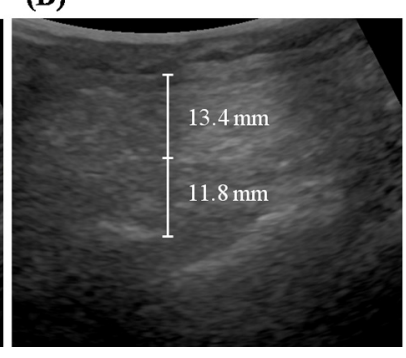

(A)

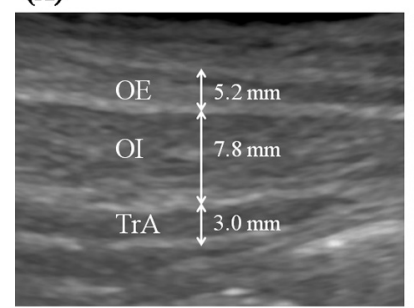

(C)

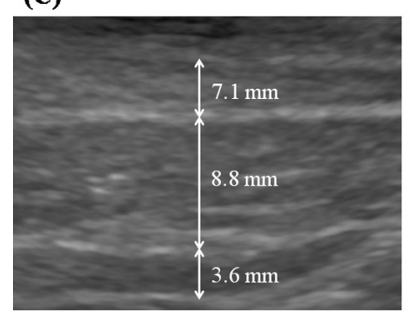

(B)

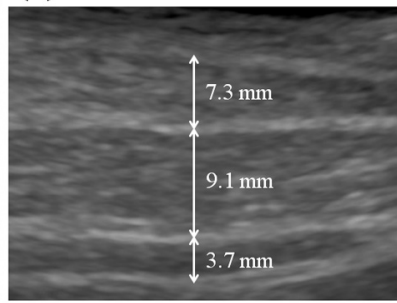

(D)

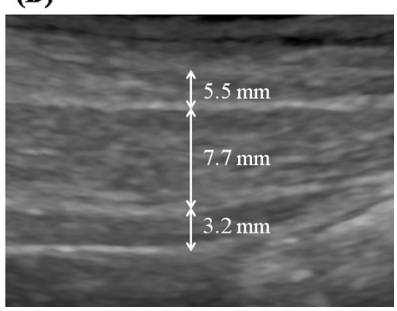

Fig. 5. Comparison of the thicknesses of three abdominal muscles (A) at rest, (B) protocol A; stimulation of abdominal muscles, (C) protocol $\mathrm{A}+\mathrm{B}$; concurrent stimulation of abdominal and lumbar muscles, (D) protocol B; stimulation of lumbar muscles

OE: obliquus externus muscle, OI: obliquus internus muscle, $\operatorname{TrA}$ : transversus abdominis muscle

(A) at rest, (B) during protocol B; stimulation of lumbar muscles, (C) during protocol $\mathrm{A}+\mathrm{B}$; concurrent stimulation of abdominal and lumbar muscles, and (D) during protocol A; stimulation of abdominal muscles

SM: superficial lumbar multifidus muscle, DM: deep lumbar multifidus muscle 
stabilizers $^{13-15)}$, and thus, extensive LM muscle atrophy is a characteristic of LDK patients ${ }^{33)}$. For these reasons, strengthening of the deep lumbar stabilizers of LDK patients is an essential component of treatment. However, specific exercises for the deep lumbar stabilizers are labor-intensive, are often difficult to maintain, and require repeated patient education sessions by skilled physical therapists. Stevens et al. ${ }^{34)}$ assessed the effects of stabilization exercises on LM muscle activation using surface electromyography, and found there was no change in LM muscle activation levels. Koumantakis et al. ${ }^{35}$ ) investigated the effects of stabilization and general exercises on the LM muscle of LBP patients and also found they had no effect on the LM muscle. They concluded that isolated LM muscle contraction is difficult to achieve and maintain during exercises ${ }^{34,35)}$. Bilgin et al. ${ }^{22}$ investigated the effect of an abdominal hollowing exercise on the LM muscle and dound it had no effect on LM muscle activation. They also reported that patients had difficulty learning and maintaining abdominal hollowing, and concluded that insufficient contraction of the LM muscle probably contributed to their negative result. They suggested NMES while performing lumbar stabilizing exercises might help the re-education of LM and TrA muscles. Therefore, active utilization of NMES is recommended for the patients with LDK in order to compensate for the limitations of deep lumbar stabilizing exercises in the enhancement of muscle-strengthening effects.

Table 1. Thicknesses ( $\mathrm{mm})$ at rest and during NMES

\begin{tabular}{lcccc}
\hline \multirow{2}{*}{ Muscle } & Rest & \multicolumn{3}{c}{ NMES protocol } \\
\cline { 3 - 5 } & & $\mathrm{A}$ & $\mathrm{B}$ & $\mathrm{A}+\mathrm{B}$ \\
\hline \multirow{2}{*}{$\mathrm{SM}$} & 12.7 & $13.1^{*}$ & $13.9^{*}$ & $13.9^{*}$ \\
& $(2.1)$ & $(1.6)$ & $(2.0)$ & $(1.8)$ \\
$\mathrm{NM}$ & 11.2 & $11.4^{*}$ & $12.2^{*}$ & $12.3^{*}$ \\
& $(1.6)$ & $(2.1)$ & $(2.0)$ & $(2.3)$ \\
$\mathrm{OE}$ & 5.2 & $7.3^{*}$ & 5.5 & $7.1^{*}$ \\
& $(2.1)$ & $(2.9)$ & $(2.1)$ & $(3.0)$ \\
OI & 7.8 & $9.1^{*}$ & 7.7 & $8.8^{*}$ \\
& $(1.8)$ & $(2.1)$ & $(1.5)$ & $(2.1)$ \\
TrA & 3.0 & $3.7^{*}$ & 3.2 & $3.7^{*}$ \\
& $(0.8)$ & $(1.1)$ & $(0.7)$ & $(1.0)$ \\
\hline
\end{tabular}

Values are means ( \pm standard deviations)

NMES: neuromuscular electrical stimulation

A: stimulation of abdominal muscles, B: stimulation of lumbar muscles, A+B: simultaneous stimulation of abdominal and lumbar muscles

SM: superficial lumbar mutifidus muscle, DM: deep lumbar mutifidus muscle, OE: obliquus externus muscle, OI: obliquus internus muscle, TrA: transversus abdominis muscle

*Significantly increased compared to the resting state, $\mathrm{p}<0.05$

Table 2. Thickness (mm) changes from rest induced by NMES

\begin{tabular}{|c|c|c|c|c|c|c|}
\hline \multirow{2}{*}{ Muscle } & \multicolumn{3}{|c|}{ NMES protocol } & \multicolumn{3}{|c|}{ Stimulation effect } \\
\hline & A (a) & $\mathrm{B}(\mathrm{b})$ & $\mathrm{A}+\mathrm{B}(\mathrm{c})$ & $\mathrm{Pa} \times \mathrm{b}$ & $\mathrm{Pa} \times \mathrm{c}$ & $\mathrm{Pb} \times \mathrm{c}$ \\
\hline $\mathrm{SM}^{\dagger}$ & $\begin{array}{c}0.5 \\
(1.1)\end{array}$ & $\begin{array}{c}1.2 \\
(1.0)\end{array}$ & $\begin{array}{c}1.3 \\
(1.1)\end{array}$ & $0.034^{\Delta}$ & $0.036^{\Delta}$ & 0.499 \\
\hline $\mathrm{DM}^{\dagger}$ & $\begin{array}{c}0.3 \\
(0.8)\end{array}$ & $\begin{array}{c}1.0 \\
(0.9)\end{array}$ & $\begin{array}{c}1.1 \\
(1.3)\end{array}$ & $0.038^{\Delta}$ & $0.028^{\Delta}$ & 0.494 \\
\hline $\mathrm{OE}^{\dagger}$ & $\begin{array}{c}2.1 \\
(1.7)\end{array}$ & $\begin{array}{l}-0.1 \\
(0.8)\end{array}$ & $\begin{array}{c}1.9 \\
(1.9)\end{array}$ & $0.002^{\Delta}$ & 0.469 & $0.005^{\Delta}$ \\
\hline $\mathrm{OI}^{\dagger}$ & $\begin{array}{c}1.3 \\
(1.1)\end{array}$ & $\begin{array}{c}0.2 \\
(0.4)\end{array}$ & $\begin{array}{c}1.0 \\
(1.2)\end{array}$ & $0.000^{\Delta}$ & 0.297 & $0.002^{\Delta}$ \\
\hline $\operatorname{Tr} \mathrm{A}^{\dagger}$ & $\begin{array}{c}0.7 \\
(0.7)\end{array}$ & $\begin{array}{c}0.7 \\
(0.7)\end{array}$ & $\begin{array}{c}0.6 \\
(0.7)\end{array}$ & $0.018^{\Delta}$ & 0.475 & $0.034^{\Delta}$ \\
\hline
\end{tabular}

Values are means ( \pm standard deviations)

NMES: neuromuscular electrical stimulation

A: stimulation of abdominal muscles, B: stimulation of lumbar muscles, A+B: simultaneous stimulation of abdominal and lumbar muscles

SM: superficial lumbar mutifidus muscle, DM: deep lumbar mutifidus muscle, OE: obliquus externus muscle, OI: obliquus internus muscle, TrA: transversus abdominis muscle

${ }^{\dagger}$ Significant difference among the A, B and A+B protocols $(\mathrm{p}<0.05)$

${ }^{\Delta} \mathrm{p}<0.05$ 
The optimal stimulation protocol for the activation of deep lumbar stabilizing muscles has not been determined. In this study, significant thickness changes in LM muscles were found during abdominal wall stimulation (protocol A). This thickness change may be the result of an indirect tensile effect of the lumbar fascia straining during contraction of the abdominal muscles ${ }^{27}$, 28). Baek et al. ${ }^{27)}$ reported LM muscle activation without lumbar region activation during NMES applied to the abdominal wall of healthy subjects. Baek et al. ${ }^{28)}$ also reported TrA and OI activations during NMES applied to the lumbar region without abdominal wall stimulation in healthy subjects. Therefore, it was our initial expectation that thickness changes in the LM and abdominal (TrA and $\mathrm{OI}$ ) muscles during protocol $\mathrm{A}+\mathrm{B}$ would be greater than during protocols $\mathrm{A}$ or $\mathrm{B}$. However, no significant difference was observed between protocols $B$ and $A+B$, or between $A$ and $A+B$. We reason these findings were due to the indirect tensile effect of the lumbar fascia as mentioned above.

The results of this study demonstrate that protocol A+B was effective at activating all the studied deep lumbar stabilizing muscles (LM, TrA and OI). Therefore, placing NMES electrodes on the 4 abdominal wall and 4 lumbar regions is suggested for the training of deep lumbar stabilizing muscles of patients with LDK. To our knowledge, no previous report has mentioned the concurrent stimulation of abdominal and back muscles to achieve contractions of the deep lumbar stabilizing muscles.

Some limitations of this study warrant consideration. First, the sample size was relatively small, and thus, further largerscale clinical studies should be conducted to quantify deep lumbar stabilizing muscle responses in NMES training. Second, RUSI is a powerful anatomic imaging tool that can demonstrate gross architecture, but the power generated by both the abdominal wall and lumbar region stimulation might be greater than the maximal voluntary contraction (MVC) detectable by RUSI. Studies using fine wire EMG may provide a more detailed view of the contributions of each stimulation protocol. Clinical studies, such as, gait analysis, are required to assess dynamic sagittal imbalance improvements before, during and after NMES of deep lumbar stabilizing muscles of patients with LDK.

In conclusion, lumbar stabilizing muscles of patients with LDK were significantly activated by NMES. Protocol A+B maximally stimulated all the studied deep spinal stabilizing muscles (LM, TrA and OI) as evidenced by RUSI. The protocol A+B may aid the development of practical NMES systems for LDK patients who suffer from postural deformity and LBP.

\section{ACKNOWLEDGEMENT}

This work was supported by the 2013 Yeungnam University Research Grant.

\section{REFERENCES}

1) Takemitsu Y, Harada Y, Iwahara T, et al.: Lumbar degenerative kyphosis. Clinical, radiological and epidemiological studies. Spine, 1988, 13: 1317-1326. [Medline] [CrossRef]

2) Lee CS, Lee CK, Kim YT, et al.: Dynamic sagittal imbalance of the spine in degenerative flat back: significance of pelvic tilt in surgical treatment. Spine, 2001, 26: 2029-2035. [Medline] [CrossRef]

3) Kang CH, Shin MJ, Kim SM, et al.: MRI of paraspinal muscles in lumbar degenerative kyphosis patients and control patients with chronic low back pain. Clin Radiol, 2007, 62: 479-486. [Medline] [CrossRef]

4) Booth KC, Bridwell KH, Lenke LG, et al.: Complications and predictive factors for the successful treatment of flatback deformity (fixed sagittal imbalance). Spine, 1999, 24: 1712-1720. [Medline] [CrossRef]

5) Farfan HF, Huberdeau RM, Dubow HI: Lumbar intervertebral disc degeneration: the influence of geometrical features on the pattern of disc degeneration — a post mortem study. J Bone Joint Surg Am, 1972, 54: 492-510. [Medline]

6) Bernhardt M, Bridwell KH: Segmental analysis of the sagittal plane alignment of the normal thoracic and lumbar spines and thoracolumbar junction. Spine, 1989, 14: 717-721. [Medline] [CrossRef]

7) Cho I, Jeon C, Lee S, et al.: Effects of lumbar stabilization exercise on functional disability and lumbar lordosis angle in patients with chronic low back pain. J Phys Ther Sci, 2015, 27: 1983-1985. [Medline] [CrossRef]

8) Panjabi MM: The stabilizing system of the spine. Part I. Function, dysfunction, adaptation, and enhancement. J Spinal Disord, 1992, 5: 383-389, discussion 397. [Medline] [CrossRef]

9) Panjabi MM: The stabilizing system of the spine. Part II. Neutral zone and instability hypothesis. J Spinal Disord, 1992, 5: 390-396, discussion 397. [Medline] [CrossRef]

10) Lee SW, Kim SY: Effects of hip exercises for chronic low-back pain patients with lumbar instability. J Phys Ther Sci, 2015, 27: 345-348. [Medline] [CrossRef]

11) Cho M, Jeon $\mathrm{H}$ : The effects of bridge exercise on an unstable base of support on lumbar stability and the thickness of the transversus abdominis. J Phys Ther Sci, 2013, 25: 733-736. [Medline] [CrossRef]

12) MacDonald D, Moseley GL, Hodges PW: Why do some patients keep hurting their back? Evidence of ongoing back 
muscle dysfunction during remission from recurrent back pain. Pain, 2009, 142: 183-188. [Medline] [CrossRef]

13) Hodges PW, Richardson CA: Altered trunk muscle recruitment in people with low back pain with upper limb movement at different speeds. Arch Phys Med Rehabil, 1999, 80: 1005-1012. [Medline] [CrossRef]

14) Butcher SJ, Craven BR, Chilibeck PD, et al.: The effect of trunk stability training on vertical takeoff velocity. J Orthop Sports Phys Ther, 2007, 37: 223-231. [Medline] [CrossRef]

15) Danneels LA, Coorevits PL, Cools AM, et al.: Differences in electromyographic activity in the multifidus muscle and the iliocostalis lumborum between healthy subjects and patients with sub-acute and chronic low back pain. Eur Spine J, 2002, 11: 13-19. [Medline] [CrossRef]

16) Hides JA, Stanton WR, McMahon S, et al.: Effect of stabilization training on multifidus muscle cross-sectional area among young elite cricketers with low back pain. J Orthop Sports Phys Ther, 2008, 38: 101-108. [Medline] [CrossRef]

17) O'Sullivan PB, Phyty GD, Twomey LT, et al.: Evaluation of specific stabilizing exercise in the treatment of chronic low back pain with radiologic diagnosis of spondylolysis or spondylolisthesis. Spine, 1997, 22: 2959-2967. [Medline] [CrossRef]

18) Lee S, Park J, Lee D: Effects of bridge exercise performed on an unstable surface on lumbar stabilizing muscles according to the knee angle. J Phys Ther Sci, 2015, 27: 2633-2635. [Medline] [CrossRef]

19) Gong W: The effect of bridge exercise accompanied by the abdominal drawing-in maneuver on an unstable support surface on the lumbar stability of normal adults. J Phys Ther Sci, 2015, 27: 47-50. [Medline] [CrossRef]

20) Hwangbo G, Lee CW, Kim SG, et al.: The effects of trunk stability exercise and a combined exercise program on pain, flexibility, and static balance in chronic low back pain patients. J Phys Ther Sci, 2015, 27: 1153-1155. [Medline] [CrossRef]

21) Kang DH, Jeon JK, Lee JH: Effects of low-frequency electrical stimulation on cumulative fatigue and muscle tone of the erector spinae. J Phys Ther Sci, 2015, 27: 105-108. [Medline] [CrossRef]

22) Bilgin $\mathrm{S}$, Temucin $\mathrm{CM}$, Nurlu G, et al.: Effects of exercise and electrical stimulation on lumbar stabilization in asymptomatic subjects: a comparative study. J Back Musculoskeletal Rehabil, 2013, 26: 261-266. [Medline]

23) Oghlan S, Crowe L, McCarthyPersson U, et al.: Electrical muscle stimulation for deep stabilizing muscles in abdominal wall. Conf Proc IEEE Eng Med Biol Soc, 2008, 2008: 2756-2759.

24) Coghlan S, Crowe L, McCarthypersson U, et al.: Neuromuscular electrical stimulation training results in enhanced activation of spinal stabilizing muscles during spinal loading and improvements in pain ratings. Conf Proc IEEE Eng Med Biol Soc, 2011, 2011: 7622-7625.

25) Miura M, Seki K, Ito O, et al.: Electrical stimulation of the abdomen preserves motor performance in the inactive elderly: a randomized controlled trial. Tohoku J Exp Med, 2012, 228: 93-101. [Medline] [CrossRef]

26) Glaser JA, Baltz MA, Nietert PJ, et al.: Electrical muscle stimulation as an adjunct to exercise therapy in the treatment of nonacute low back pain: a randomized trial. J Pain, 2001, 2: 295-300. [Medline] [CrossRef]

27) Baek SO, Cho HK, Jung GS, et al.: Verification of an optimized stimulation point on the abdominal wall for transcutaneous neuromuscular electrical stimulation for activation of deep lumbar stabilizing muscles. Spine J, 2014, 14: 2178-2183. [Medline] [CrossRef]

28) Baek SO, Ahn SH, Jones R, et al.: Activations of deep lumbar stabilizing muscles by transcutaneous neuromuscular electrical stimulation of lumbar paraspinal regions. Ann Rehabil Med, 2014, 38: 506-513. [Medline] [CrossRef]

29) Ferreira PH, Ferreira ML, Hodges PW: Changes in recruitment of the abdominal muscles in people with low back pain: ultrasound measurement of muscle activity. Spine, 2004, 29: 2560-2566. [Medline] [CrossRef]

30) Koppenhaver SL, Fritz JM, Hebert JJ, et al.: Association between history and physical examination factors and change in lumbar multifidus muscle thickness after spinal manipulation in patients with low back pain. J Electromyogr Kinesiol, 2012, 22: 724-731. [Medline] [CrossRef]

31) Kim WJ, Kang JW, Kang SI, et al.: Factors affecting clinical results after corrective osteotomy for lumbar degenerative kyphosis. Asian Spine J, 2010, 4: 7-14. [Medline] [CrossRef]

32) Enomoto M, Ukegawa D, Sakaki K, et al.: Increase in paravertebral muscle activity in lumbar kyphosis patients by surface electromyography compared with lumbar spinal canal stenosis patients and healthy volunteers. J Spinal Disord Tech, 2012, 25: E167-E173. [Medline] [CrossRef]

33) Hasue M, Fujiwara M, Kikuchi S: A new method of quantitative measurement of abdominal and back muscle strength. Spine, 1980, 5: 143-148. [Medline] [CrossRef] 
34) Stevens VK, Bouche KG, Mahieu NN, et al.: Trunk muscle activity in healthy subjects during bridging stabilization exercises. BMC Musculoskelet Disord, 2006, 7: 75. [Medline] [CrossRef]

35) Koumantakis GA, Watson PJ, Oldham JA: Supplementation of general endurance exercise with stabilisation training versus general exercise only. Physiological and functional outcomes of a randomised controlled trial of patients with recurrent low back pain. Clin Biomech (Bristol, Avon), 2005, 20: 474-482. [Medline] [CrossRef] 